\title{
Topological Invariance under Line Graph Transformations
}

\author{
Allen D. Parks \\ Electromagnetic and Sensor Systems Department, Naval Surface Warfare Center Dahlgren Division, \\ 18444 Frontage Road Suite 327, Dahlgren, VA 22448-5161, USA; E-Mail: allen.parks@navy.mil
}

Received: 6 April 2012; in revised form: 1 June 2012 / Accepted: 4 June 2012 /

Published: 8 June 2012

\begin{abstract}
It is shown that the line graph transformation $G \mapsto L(G)$ of a graph $G$ preserves an isomorphic copy of $G$ as the nerve of a finite simplicial complex $\boldsymbol{K}$ which is naturally associated with the Krausz decomposition of $L(G)$. As a consequence, the homology of $\boldsymbol{K}$ is isomorphic to that of $G$. This homology invariance algebraically confirms several well known graph theoretic properties of line graphs and formally establishes the Euler characteristic of $G$ as a line graph transformation invariant.
\end{abstract}

Keywords: algebraic graph theory; line graph; Krausz decomposition; homology; graph invariant; Euler characteristic

\section{Introduction}

Because of its intrinsic interest, the line graph transformation $G \mapsto L(G)$ of a graph $G$ has been widely studied. The impetus for much of this research was provided by Ore's discussion of line graphs and problems associated with them [1]. Line graphs are also interesting from a practical standpoint, since it has been shown that certain NP-complete problems for graphs are polynomial time problems for line graphs, e.g., [2]. Because of their utility for recognizing non-isomorphic graphs, graph invariants have also been the object of intensive research, e.g., [3].

In this paper, a new topological invariance associated with the line graph transformation is found using the natural relationship between a Krausz decomposition of $L(G)$ and an abstract simplicial complex $\boldsymbol{K}$. In particular, it is shown that, under the line graph transformation, an isomorphic copy of $G$ is preserved as the nerve of $\boldsymbol{K}$. As a consequence, the homology of $G$ is isomorphic to that of $\boldsymbol{K}$ and an application of the Euler-Poincare formula yields the Euler characteristic of $G$ as a line graph transformation invariant. This invariance also algebraically confirms several well-known graph theoretic properties of line graphs. 
The remainder of this paper is organized as follows: The relevant definitions and terminology are summarized in the next section. Required preliminary lemmas are provided in Section 3 and the main results are established in Section 4. A simple illustrative example is presented in Section 5. Closing remarks comprise the final section of this paper.

\section{Definitions and Terminology}

A graph $G$ is a pair $(V(G), E(G))$, where $V(G)$ is a finite non-empty set of vertices and $E(G)$ is a set of doubleton subsets of $V(G)$ called edges. $G$ is a non-empty graph when $V(G) \neq \emptyset \neq E(G)$ and $G$ is a $(p, q)$ graph if $|V(G)|=p$ and $|E(G)|=q$. A $(1,0)$ graph is a trivial graph. The number of edges incident to a vertex $v$ is the valency of $v$. A vertex of valency zero is an isolated vertex (only graphs without isolated vertices are considered here). A component of $G$ is a maximally connected subgraph of $G$. A complete graph $K_{n}$ on $n$ vertices has every pair of vertices adjacent. When $V(G)$ is partitioned into two sets $V_{1}$ and $V_{2}$ of cardinality $m$ and $n$ such that each vertex in $V_{1}$ is adjacent to every vertex in $V_{2}$, then $G$ is the complete bipartite graph $K_{m, n}$. If $G$ is connected and has no cycles, then $G$ is a tree. Graph $G_{1}$ is isomorphic to graph $G_{2}$ (denoted $G_{1} \cong G_{2}$ ) if there is an adjacency preserving bijective map $\varphi: V\left(G_{1}\right) \rightarrow V\left(G_{2}\right)$.

Associate with any non-empty graph $G$ its line graph $L(G)$ which has $E(G)$ as its vertex set and has as its edge set those pairs in $E(G)$ which are adjacent in $G$. A collection $\mathcal{K}$ of subgraphs of a graph $F$ is a Krausz decomposition of $F$ if (i) each member of $\mathcal{K}$ is a complete graph; (ii) every edge of $F$ is in exactly one member of $\mathcal{K}$; and (iii) every vertex of $F$ is in exactly two members of $\mathcal{K}$. A nonempty graph is a line graph if, and only if, it has a Krausz decomposition and-provided that $G_{1}$ and $G_{2}$ are non-trivial connected graphs- $L\left(G_{1}\right) \cong L\left(G_{2}\right)$ if, and only if, $G_{1} \cong G_{2}$ or $\left\{G_{1}, G_{2}\right\}$ is (up to isomorphism) the unordered pair $\left\{K_{3}, K_{1,3}\right\}$ [4].

A hypergraph is a pair $(\mathcal{N}, \mathcal{E})$, where $\mathcal{N}$ is a finite set of vertices and $\mathcal{E}$ is a set of hyperedges which are non-empty subsets of $\mathcal{N}$. A Krausz hypergraph $\mathcal{H}$ of a line graph $L(G)$ has $V(L(G)$ ) as its vertex set and the family of subsets of $V(L(G))$ that induce the members of $\mathcal{K}$ as its hyperedges.

If $S$ is a finite set, then the closure $C l(S)$ of $S$ is the family of non-empty subsets of $S$. The closure $C l(\mathcal{H})$ of $\mathcal{H}$ is the union of the closures of its hyperedges, i.e., $C l(\mathcal{H})=\bigcup_{E} \in \mathcal{E} C l(E)$. The number of sets of cardinality $k$ in $C l(\mathcal{H})$ is $h_{k}$ and $\xi$ is the maximum $k$ for which $h_{k} \neq 0$.

Let $\left\{a_{0}, \ldots, a_{k}\right\}$ be a set of geometrically independent points in $\mathbb{R}^{n}$. The k-simplex (or simplex) $\sigma^{k}$ spanned by $\left\{a_{0}, \ldots, a_{k}\right\}$ is the set of points $x \in \mathbb{R}^{n}$ for which there exist non-negative real numbers $\lambda_{0}, \ldots, \lambda_{k}$ such that $x=\sum_{0} \leq i \leq k \lambda_{i} a_{i}$ and $\sum_{0 \leq i \leq k} \lambda_{i}=1$. In this case $\left\{a_{0}, \ldots, a_{k}\right\}$ is the vertex set of $\sigma^{k}$. A face of $\sigma^{k}$ is any simplex spanned by a non-empty subset of $\left\{a_{0}, \ldots, a_{k}\right\}$. A finite geometric simplicial complex (or complex) $\boldsymbol{K}$ is a finite union of simplices such that: $(i)$ every face of a simplex of $\boldsymbol{K}$ is in $\boldsymbol{K}$; and (ii) the non-empty intersection of any two simplices of $\boldsymbol{K}$ is a common face of each. Here it is assumed that all simplicial complexes are finite. Consequently, the dimension of $\boldsymbol{K}$ is the largest positive integer $m$ such that $\boldsymbol{K}$ contains an $m$-simplex. The vertex scheme of $\boldsymbol{K}$ is the family of all vertex sets which span the simplices of $\boldsymbol{K}$. The $n$-skeleton of $\boldsymbol{K}$ is the set of all simplices in $\boldsymbol{K}$ with dimension $\leq n$. $\boldsymbol{K}$ is connected if, and only if, its 1 -skeleton is connected. If $\left\{L_{i}\right\}$ is a family of subcomplexes of $\boldsymbol{K}$, then $\bigcup_{i} L_{i}$ and $\bigcap_{i} L_{i} \neq \emptyset$ are subcomplexes of $\boldsymbol{K}$. 
A finite abstract simplicial complex (or abstract complex) is a finite family $\boldsymbol{S}$ of finite non-empty sets such that if $A$ is in $\boldsymbol{S}$, then so is every non-empty subset of $A$. Thus, the vertex scheme of a complex is an abstract complex as are finite unions of set closures and finite intersections of set closures when they are non-empty.

Two abstract complexes $\boldsymbol{S}$ and $\boldsymbol{T}$ are isomorphic if there is a bijection $\varphi$ from the vertex set of $\boldsymbol{S}$ onto the vertex set of $\mathcal{T}$ such that $\left\{a_{0}, \ldots, a_{k}\right\} \in \mathcal{S}$ if, and only if, $\left\{\varphi\left(a_{0}\right), \ldots, \varphi\left(a_{k}\right)\right\} \in \mathcal{T}$. Every abstract complex $\boldsymbol{S}$ is isomorphic to the vertex scheme of some geometric simplicial complex $\boldsymbol{K}$-in which case $\boldsymbol{K}$ is the geometric realization of $\boldsymbol{S}$ and is uniquely determined (up to linear isomorphism). An isomorphism between $\boldsymbol{S}$ and the vertex scheme of $\boldsymbol{K}$ is denoted $\boldsymbol{S} \simeq \boldsymbol{K}$.

To each simplicial complex $\boldsymbol{K}$ there corresponds a chain complex, i.e., abelian groups $\mathcal{C}_{p}(\boldsymbol{K})$ and homomorphisms $\partial_{p+1}: \mathcal{C}_{p+1}(\boldsymbol{K}) \rightarrow \mathcal{C}_{p}(\boldsymbol{K}), p \geq 0$. If $\boldsymbol{K}$ is finite and $\eta_{p}(\boldsymbol{K})$ is the number of $p$-simplices in $\boldsymbol{K}$, then the rank of $\mathcal{C}_{p}(\boldsymbol{K})$ is $\eta_{p}(\boldsymbol{K})$ and $\mathcal{C}_{p}(\boldsymbol{K})$ is isomorphic to (here $\cong$ denotes both group and graph isomorphism) the direct sum of $\eta_{p}(\boldsymbol{K})$ copies of the additive group of integers $\mathbb{Z}$. The $p^{\text {th }}$ homology group of $\boldsymbol{K}$ is the quotient group $H_{p}(\boldsymbol{K}) \equiv \operatorname{ker} \partial_{p} /$ im $\partial_{p+1}$ and its rank is the $p^{\text {th }}$ betti number $b_{p}(\boldsymbol{K})$. Complexes $\boldsymbol{K}$ and $\boldsymbol{K}^{\prime}$ are homologically isomorphic when $H_{p}(\boldsymbol{K}) \cong H_{p}\left(\boldsymbol{K}^{\prime}\right), p \geq 0$, and $\boldsymbol{K}$ is homologically acyclic (or acyclic) if $H_{p}(\boldsymbol{K}) \cong 0, p \geq 1$. The complex of a simplex is acyclic and if $\boldsymbol{K}$ is empty, then $\boldsymbol{K}$ is acyclic. The number of components of $\boldsymbol{K}$ is the betti number $b_{0}(\boldsymbol{K})$.

A cover of a simplicial complex $\boldsymbol{K}$ is a family of subcomplexes $\mathcal{L}=\left\{L_{\alpha}: \alpha \in A\right\}$ with $\boldsymbol{K}=\bigcup_{\alpha} L_{\alpha}$, where $A$ is an index set. The family $\mathcal{L}$ is an acyclic cover if each $L_{\alpha}$ and each finite intersection $\bigcap_{\alpha} L_{\alpha}$ are acyclic. The nerve $N(\mathcal{L})$ of $\mathcal{L}$ is the simplicial complex having $A$ as its vertex set with $\Delta=\left\{\alpha_{0}, \ldots, \alpha_{n}\right\}$ a simplex in $N(\mathcal{L})$ if $\bigcap_{\Delta} L_{\alpha} \neq \emptyset$.

\section{Preliminary Lemmas}

The following lemmas are required to prove the main results in the next section. The first four are well known and are stated without proof for completeness.

Lemma 1. [5] (Euler-Poincaré) If $K$ is a complex of dimension $m$, then

$$
\sum_{0 \leq p \leq m}(-1)^{p} \eta_{p}(K)=\sum_{0 \leq p \leq m}(-1)^{p} b_{p}(K)
$$

Lemma 2. [6] A non-empty connected graph $G$ is a tree if, and only if, $G$ is homologically acyclic and $H_{0}(G) \cong \mathbb{Z}$.

Lemma 3. [7] Let $F$ be a graph. Then $F \cong L(G)$ for some graph $G$ if, and only if, the vertices of $G$ can be placed into one-to-one correspondence with the members of a Krausz decomposition $\mathcal{K}$ of $F$ such that two vertices of $G$ are adjacent if, and only if, the corresponding members of $\mathcal{K}$ have a common vertex.

Lemma 4. [8] (Folkman-Leray) If $\mathcal{L}$ is an acyclic cover of a simplicial complex $\boldsymbol{K}$, then $\boldsymbol{K}$ and $N(\mathcal{L})$ are homologically isomorphic.

The closure operation $C l$ is important for proving the main results of this paper. The required key properties of $\mathrm{Cl}$ are provided by the next lemma. Since the proof is straight forward it is omitted. 
Lemma 5. Let $\left\{E_{m}: m \in I\right\}$ be a collection of non-empty finite sets. Then the following statements are true:

(1) $\bigcap_{m} \operatorname{Cl}\left(E_{m}\right)=\operatorname{Cl}\left(\bigcap_{m} E_{m}\right)$;

(2) $A \cap B \neq \emptyset \Leftrightarrow C l(A) \cap C l(B) \neq \emptyset$; and

(3) $A=\emptyset \Leftrightarrow C l(A)=\emptyset$.

Lemma 6. Let $\mathcal{H}=(\mathcal{N}, \mathcal{E})$ be a Krausz hypergraph. Suppose $\boldsymbol{S}=C l(\mathcal{H})=\bigcup_{E} \in \mathcal{E} C l(E)$ is the abstract complex associated with $\mathcal{H}$ and its geometric realization is the Krausz complex $\boldsymbol{K}$. If $E_{i}, E_{j}, E_{k} \in \mathcal{E}$ are distinct and the subcomplex $K_{i}$ of $\mathbf{K}$ corresponds to the abstract complex $C l\left(E_{i}\right)$ of $\boldsymbol{S}$, then

(1) $\left|K_{i} \cap K_{j}\right| \leq 1$; and

(2) $K_{i} \cap K_{j} \cap K_{k}=\emptyset$.

Proof. Condition (1) follows since $E_{i}$ and $E_{j}$ are induced by a Krausz decomposition and have at most one vertex in common (apply (2) of Lemma 5 with $A=E_{i}$ and $B=E_{j}$ ). Condition (2) follows since no three hyperedges of $\mathcal{H}$ have a common vertex (apply (3) of Lemma 5 with $A=E_{i} \cap E_{j} \cap E_{k}=\emptyset$ and then (1) of Lemma 5 with $\bigcap_{m} E_{m}=E_{i} \cap E_{j} \cap E_{k}$ ).

\section{Main Results}

The terminology and results of the previous sections are now used to prove the following main results of this paper. In what follows, it is assumed that: $(i) \boldsymbol{K}$ is a Krausz complex associated with a Krausz hypergraph $\mathcal{H}=(\mathcal{N}, \mathcal{E})$ of a graph $F \cong L(G)$; (ii) $G$ is a non-trivial connected graph; (iii) $G$ is not isomorphic to $K_{3}$ or $K_{1,3}$; and (iv) $\mathcal{L}=\left\{K_{i} \subset \boldsymbol{K}: K_{i} \cong C l\left(E_{i}\right), E_{i} \in \mathcal{E}\right\}$.

Theorem 1. $G \cong N(\mathcal{L})$.

Proof. By definition of $\mathcal{L},|\mathcal{L}|=|\mathcal{E}|$. Also, $K_{i} \cap K_{j} \neq \emptyset$ if, and only if, the corresponding pair of hyperedges in $\mathcal{H}$ have a common vertex. Since $F \cong L(G)$, then Lemma 3 yields a correspondence between $V(G)$ and $\mathcal{E}$ such that $u$ adjacent to $v$ in $G$ if, and only if, corresponding hyperedges have a vertex in common. Therefore, it follows from the definition of nerve that $G \cong N(\mathcal{L})$. (Recall that here $G$ is assumed to not be isomorphic to $K_{3}$ or $K_{1,3}$ ).

Lemma 7. $\mathcal{L}$ is an acyclic cover of $\boldsymbol{K}$.

Proof. By definition $\boldsymbol{K}=\bigcup_{i} K_{i}$, where each $K_{i}$ is the complex of a simplex. Thus, $\mathcal{L}$ covers $\boldsymbol{K}$. Since each $K_{i}$ and (via Lemma 6) every finite intersection of the $K_{i}$ 's is acyclic, then $\mathcal{L}$ is an acyclic cover of $\boldsymbol{K}$.

Theorem 2. $\mathrm{K}$ and $\mathrm{G}$ are homologically isomorphic.

Proof. $\boldsymbol{K}$ and $N(\mathcal{L})$ are homologically isomorphic (Lemma 7 and Lemma 4). Since $G \cong N(\mathcal{L})$ (Theorem 1), then $\boldsymbol{K}$ and $G$ are also homologically isomorphic.

Corollary 1. If $G$ is a $(p, q)$ graph and $F \cong L(G)$, then

$$
\sum_{1 \leq k \leq \xi}(-1)^{k-1} h_{k}=p-q
$$


Proof. The left hand side of the Euler-Poincaré Formula (1), first for $K=\boldsymbol{K}$ and then for $K=G$, may be equated since, from Theorem 2, the corresponding right hand sides are equal. But when $G=K$, the left hand side of Equation (1) is $p-q$. Also, because of the one-to-one correspondence between the $k-1$ dimensional simplices of $\boldsymbol{K}$ and the sets of size $k$ in $\boldsymbol{S}=C l(\mathcal{H})$ it is the case that the dimension of $\boldsymbol{K}$ is $\boldsymbol{\xi}-1$ and $\boldsymbol{\eta}_{k-1}(\boldsymbol{K})=h_{k}$. The validity of Equation (2) now follows from these observations and the appropriate $K=\boldsymbol{K}$ and $K=G$ substitutions in the left hand side of Equation (1).

Corollary 2. The graph $G$ and corresponding line graph $L(G)$ have the same number of components.

Proof. From Theorem 2, $b_{0}(G)=b_{0}(\boldsymbol{K})$ so that $G$ and $\boldsymbol{K}$ have the same number of components. But $L(G)$ and $\boldsymbol{K}$ also have an identical number of components since $L(G)$ is isomorphic to the 1-skeleton of $\boldsymbol{K}$. (This implies that $L(G)$ is connected since it is assumed here that $G$ is connected).

Corollary 3. Let $L(G)$ be a connected line graph. Then $G$ is a tree if, and only if,

$$
\sum_{1 \leq k \leq \xi}(-1)^{k-1} h_{k}=1
$$

Proof. Since $L(G)$ is connected, an application of Corollary 2 shows that $G$ is connected. A connected $(p, q)$ graph $G$ is a tree if, and only if, $p-q=1$. The result follows from Equation (2).

\section{Example}

In order to illustrate the theory developed above, consider the non-trivial connected $(4,4)$ graph $G$, its line graph $L(G)$, and a Krausz decomposition of $L(G)$ shown in Figure 1. The associated Krausz hypergraph $\mathcal{H}$ has the sets $E_{1}=\{\mathrm{a}, \mathrm{b}\}, E_{2}=\{\mathrm{a}, \mathrm{d}\}, E_{3}=\{\mathrm{b}, \mathrm{c}, \mathrm{d}\}$, and $E_{4}=\{\mathrm{c}\}$ as it hyperedges. Their closures are

$$
\begin{gathered}
C l\left(E_{1}\right)=\{\{\mathrm{a}, \mathrm{b}\},\{\mathrm{a}\},\{\mathrm{b}\}\}, \\
C l\left(E_{2}\right)=\{\{\mathrm{a}, \mathrm{d}\},\{\mathrm{a}\},\{\mathrm{d}\}\}, \\
C l\left(E_{3}\right)=\{\{\mathrm{b}, \mathrm{c}, \mathrm{d}\},\{\mathrm{b}, \mathrm{c}\},\{\mathrm{b}, \mathrm{d}\},\{\mathrm{c}, \mathrm{d}\},\{\mathrm{b}\},\{\mathrm{c}\},\{\mathrm{d}\}\},
\end{gathered}
$$

and

$$
C l\left(E_{4}\right)=\{\{\mathrm{c}\}\}
$$

so that

$$
\mathcal{L}=\left\{C l\left(E_{1}\right), C l\left(E_{2}\right), C l\left(E_{3}\right), C l\left(E_{4}\right)\right\}
$$

and

$$
C l(\mathcal{H})=\{\{\mathrm{b}, \mathrm{c}, \mathrm{d}\},\{\mathrm{a}, \mathrm{b}\},\{\mathrm{a}, \mathrm{d}\},\{\mathrm{b}, \mathrm{c}\},\{\mathrm{b}, \mathrm{d}\},\{\mathrm{c}, \mathrm{d}\},\{\mathrm{a}\},\{\mathrm{b}\},\{\mathrm{c}\},\{\mathrm{d}\}\} .
$$

It is clear that $\mathcal{L}$ is an acyclic cover of the Krausz complex associated with $C l(\mathcal{H})$. 
Figure 1. The line graph of a $(4,4)$ graph and its Krausz decomposition.

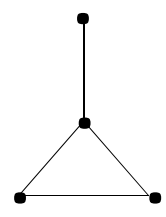

G

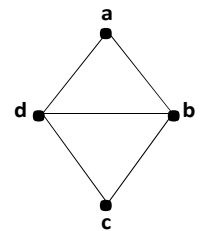

L(G)

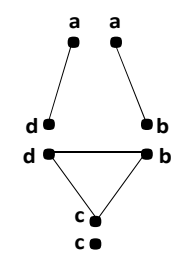

c•

The nerve $N(\mathcal{L})$ of $\mathcal{L}$ has the set $\{1,2,3,4\}$ of $\mathcal{H}$ hyperedge subscripts as its set of vertices. Since

$$
C l\left(E_{1}\right) \cap C l\left(E_{2}\right) \neq \emptyset, C l\left(E_{1}\right) \cap C l\left(E_{3}\right) \neq \emptyset, C l\left(E_{2}\right) \cap C l\left(E_{3}\right) \neq \emptyset, C l\left(E_{3}\right) \cap C l\left(E_{4}\right) \neq \emptyset
$$

and

$$
C l\left(E_{1}\right) \cap C l\left(E_{4}\right)=\emptyset, C l\left(E_{2}\right) \cap C l\left(E_{4}\right)=\emptyset
$$

then the doubleton subsets $\{1,2\},\{1,3\},\{2,3\}$, and $\{3,4\}$ are $N(\mathcal{L})$ edges (see Figure 2). It is obvious from Figure 1 and Figure 2 that - as required by Theorem $1-G \cong N(\mathcal{L})$.

Figure 2. The nerve of the acyclic cover of the Krausz complex associated with $L(G)$.

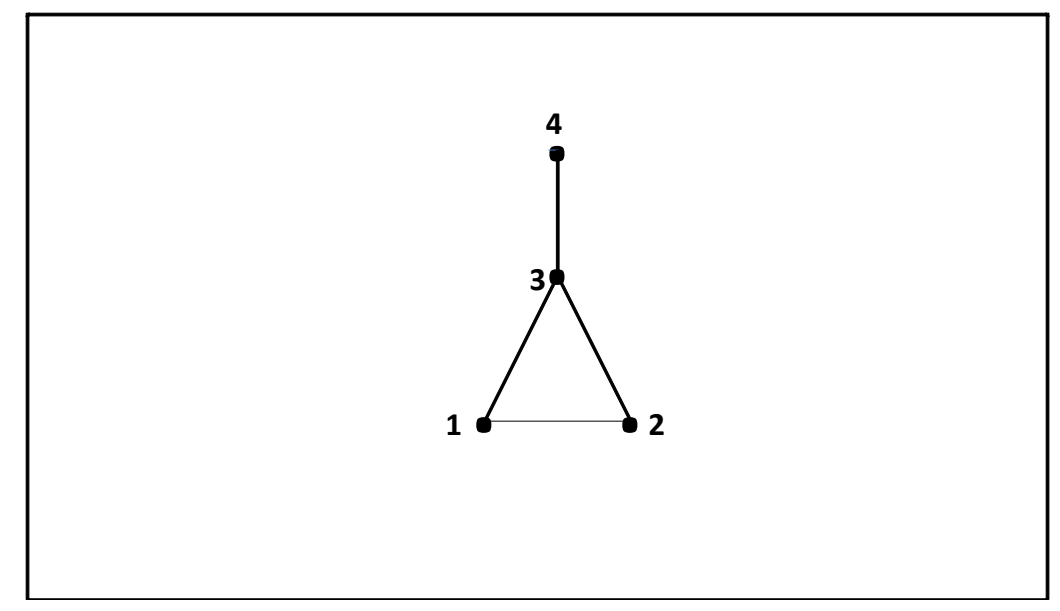

Since $G$ is a $p=4=q$ graph, then $p-q=0$. It is also readily determined from $C l(\mathcal{H})$ that $h_{3}=1$, $h_{2}=5, h_{1}=4$, and $\xi=3$, so that - as required by Corollary $1-$

$$
\sum_{1 \leq k \leq 3}(-1)^{k-1} h_{k}=4-5+1=0=p-q
$$

\section{Closing Remarks}

It has been shown that a Krausz decomposition of the line graph of a graph $G$ defines both an abstract simplicial complex and an acyclic cover $\mathcal{L}$ of a geometric realization $\boldsymbol{K}$ of the complex such that: $(i)$ the nerve of $\mathcal{L}$ is isomorphic to $G$ (i.e., the line graph transformation of $G$ preserves an isomorphic copy of $G$ as the nerve of $\mathcal{L}$ ); and (ii) $K$ and $G$ are homologically isomorphic (i.e., the line graph transformation of $G$ preserves the homology of $G$ as the homology of $\mathbf{K}$ ). Item (ii) algebraically 
confirms the graph theoretic fact that $G$ and $L(G)$ have the same number of components when $G$ has no isolated vertices. Thus, it establishes the Euler characteristic of $G$ as a line graph transformation invariant and provides Equation (3) as a condition that must be satisfied by the abstract simplicial complex $C l(\mathcal{H})$ associated with a Krausz decomposition of a line graph of $G$ when $G$ is a connected tree. It is also interesting to note that Corollary 3 is an algebraic analogue of Rao's Theorem [9].

\section{Acknowledgments}

This work was supported by a grant from the Naval Surface Warfare Center Dahlgren Division's In-house Laboratory Independent Research program.

\section{References}

1. Ore, O. Theory of Graphs; American Mathematical Society: Providence, RI, USA, 1962.

2. Lovásv, L. Vertex Packing Algorithms. In Automata, Languages, and Programming, 12th Colloquium Nafplion, Greece, 15-19 July, 1985; Brauer, W., Ed.; Springer-Verlag: Berlin, Germany, 1985; pp. 1-14.

3. Brigham, R.; Dutton, R. A compilation of relations between graph invariants. Networks 1985, 15, 73-107.

4. Hemminger, R.; Beineke, L. Line Graphs and Line Digraphs. In Selected Topics in Graph Theory; Beineke, L., Wilson, R., Eds.; Academic Press: London, UK, 1978; pp. 271-305.

5. Hocking, J.; Young, G. Topology; Addison-Wesley: Reading, MA, USA, 1961; p. 242.

6. Hu, S. Homology Theory: A First Course in Algebraic Topology; Holden-Day: San Francisco, CA, USA, 1966; p. 119.

7. Behzad, M.; Chartrand, G.; Lesniak-Foster, L. Graphs and Digraphs; Wadsworth International Group: Belmont, CA, USA, 1979; p. 190.

8. Rotman, J. An Introduction to Algebraic Topology; Springer-Verlag: New York, NY, USA, 1988; p. 154.

9. Berge, C. Graphs and Hypergraphs; North-Holland: London, UK, 1973; p. 413.

(C) 2012 by the author; licensee MDPI, Basel, Switzerland. This article is an open access article distributed under the terms and conditions of the Creative Commons Attribution license (http://creativecommons.org/licenses/by/3.0/). 